\title{
Symmetrical thalamic calcifications
}

INSERM

\section{Source}

INSERM. (1999). Orphanet: an online rare disease and orphan drug data base.

Symmetrical thalamic calcifications. ORPHA:1314

Symmetrical thalamic calcifications are clinically distinguished by a low Apg ar score,

spasticity or marked hypotonia, weak or absent cry, poor feeding, and facial diplegia or weakness. 